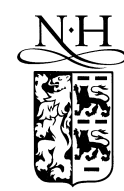

ELSEVIER

\title{
Monopole solutions in AdS space
}

\author{
A.R. Lugo ${ }^{1}$, E.F. Moreno ${ }^{1}$, F.A. Schaposnik ${ }^{2}$ \\ Departamento de Física, Universidad Nacional de La Plata, C.C. 67, 1900 La Plata, Argentina
}

Received 26 November 1999; accepted 14 December 1999

Editor: L. Alvarez-Gaumé

\begin{abstract}
We find monopole solutions for a spontaneously broken $S U(2)$-Higgs system coupled to gravity in asymptotically anti-de Sitter space. We present new analytic and numerical results discussing, in particular, how the gravitational instability of self-gravitating monopoles depends on the value of the cosmological constant. (C) 2000 Published by Elsevier Science B.V. All rights reserved.
\end{abstract}

PACS: 11.27 ; $11.15 .-\mathrm{q} ; 11.10 .-\mathrm{z}$

\section{Introduction}

Gravitating monopole solutions to gauge theories have attracted many investigations in the last 25 years [1-15]. In particular, the existence of selfgravitating monopoles in spontaneously broken nonAbelian gauge theories, their properties, relation with black hole solutions and their relevance in Cosmology have been thoroughly discussed. Most of these investigations correspond to asymptotically flat space-time but there have been recently several studies for the case in which the cosmological constant $\Lambda$ is non-vanishing [16-18]. In particular, we have discussed in [18] the existence of gravitating monopole solutions in the case in which space-time

\footnotetext{
${ }^{1}$ CONICET.

${ }^{2}$ Associate CICBA.
}

is asymptotically anti-de Sitter (AdS), which in our conventions corresponds to $\Lambda<0$. Regular (monopole and dyon) and singular (black hole) solutions have been found in this case and the properties of the magnetically charged solutions for vanishing Newton constant $G$ were analysed.

It is the purpose of the present work to complete the investigation initiated in [18] studying in detail the monopole solution in asymptotically AdS space both for vanishing and finite $G$, analytically and numerically. The plan of the paper is the following: we present in Section 2 the model, the spherically symmetric ansatz and the appropriate boundary conditions leading to gravitating monopoles and dyons. Then, in Section 3, we discuss, analytically, some relevant properties of the magnetically charged solution and then describe in detail the numerical results both for $G=0$ and $G \neq 0$. We summarize and discuss our results in Section 4. 


\section{The model}

We consider the action for SU(2) Yang-MillsHiggs theory coupled to gravity in asymptotically anti-de-Sitter space. The action is defined as $S=S_{\mathrm{G}}+S_{\mathrm{YM}}+S_{\mathrm{H}}=\int d^{D} x \sqrt{|G|}\left(L_{\mathrm{G}}+L_{\mathrm{YM}}+L_{\mathrm{H}}\right)$

with

$L_{\mathrm{G}}=\frac{1}{\alpha_{0}}\left(\frac{1}{2} R-\Lambda\right)$

$L_{\mathrm{YM}}=-\frac{1}{4 e^{2}} F_{\mu \nu}^{a} F^{a \mu \nu}$

$L_{\mathrm{H}}=-\frac{1}{2} D_{\mu} H^{a} D^{\mu} H^{a}-V(H)$

$V(H)=\frac{\lambda}{4}\left(H^{a} H^{a}-h_{0}{ }^{2}\right)^{2}$

Here $F_{\mu \nu}^{a},(a=1,2,3)$ is the field strength,

$F_{\mu \nu}^{a}=\partial_{\mu} A_{\nu}-\partial_{\nu} A_{\mu}^{a}+\varepsilon^{a b c} A_{\mu}^{b} A_{\nu}^{c}$

and the covariant derivative $D_{\mu}$ acting on the Higgs triplet $H^{a}$ is given by

$D_{\mu} H^{a}=\partial_{\mu} H^{a}+\varepsilon^{a b c} A_{\mu}^{b} H^{c}$

We have defined

$\alpha_{0} \equiv 8 \pi G$

where $G$ is the Newton constant, $e$ the gauge coupling and $\Lambda$ is the cosmological constant (with our conventions $\Lambda<0$ corresponds, in the absence of matter, to anti-de Sitter space).

The equations of motion that follow from (1) are

$E_{\mu \nu}+\Lambda G_{\mu \nu}=\alpha_{0}\left(T_{\mu \nu}^{\mathrm{YM}}+T_{\mu \nu}^{H}\right)$

$D_{\rho} D^{\rho} H^{a}=\frac{\delta V(H)}{\delta H^{a}}$

$\frac{1}{e^{2}} D^{\rho} F_{\mu \rho}^{a}=\varepsilon^{a b c}\left(D_{\mu} H^{b}\right) H^{c}$

where $E_{\mu \nu}$ is the Einstein tensor and the matter energy-momentum tensor is given by

$T_{\mu \nu}^{\mathrm{YM}}=\frac{1}{e^{2}}\left(-F_{\mu \rho}^{a} F_{a \nu}{ }^{\rho}+\frac{1}{2} G_{\mu \nu} F_{\rho \sigma}^{a} F_{a}^{\rho \sigma}\right)$

$T_{\mu \nu}^{H}=D_{\mu} H^{a} D_{\nu} H^{a}+G_{\mu \nu} L_{\mathrm{H}}$
The most general static spherically symmetric form for the metric in 3 spatial dimensions together with the 't Hooft-Polyakov-Julia-Zee ansatz for the gauge and Higgs fields in the usual vector notation reads

$$
\begin{aligned}
G= & -\mu(x) A(x)^{2} d^{2} t+\mu(x)^{-1} d^{2} r+r^{2} d^{2} \Omega_{2} \\
\boldsymbol{A}= & d \operatorname{te~}_{0} J(x) \check{e}_{r}-d \theta(1-K(x)) \check{e}_{\varphi} \\
& +d \varphi(1-K(x)) \sin \theta \check{e}_{\theta} \\
\boldsymbol{H}= & h_{0} H(x) \check{e}_{r}
\end{aligned}
$$

where we have introduced the dimensionless coordinate $x \equiv e h_{0} r$ and $h_{0}$ sets the mass scale $\left(\left[h_{0}\right]=\right.$ $\left.m^{1}\right)$.

Using this ansatz, the equations of motion take the form

$$
\begin{aligned}
& (x \mu(x))^{\prime}=1+3 \gamma_{0} x^{2} \\
& -\alpha_{0} h_{0}{ }^{2}\left(\mu(x) V_{1}+V_{2}\right. \\
& \left.+\frac{x^{2}}{2} \frac{J^{\prime}(x)^{2}}{A(x)^{2}}+\frac{J(x)^{2} K(x)^{2}}{\mu(x) A(x)^{2}}\right) \\
& x A^{\prime}(x)=\alpha_{0} h_{0}{ }^{2}\left(V_{1}+\frac{J(x)^{2} K(x)^{2}}{\mu(x)^{2} A(x)^{2}}\right) A(x) \\
& \left(\mu(x) A(x) K^{\prime}(x)\right)^{\prime} \\
& =A(x) K(x)\left(\frac{K(x)^{2}-1}{x^{2}}\right. \\
& \left.+H(x)^{2}-\frac{J(x)^{2}}{\mu(x) A(x)^{2}}\right) \\
& =A(x) H(x)\left(2 K(x)^{2}+\frac{\lambda}{e^{2}} x^{2}\left(H(x)^{2}-1\right)\right)
\end{aligned}
$$

$\mu(x)\left(\frac{x^{2} J^{\prime}(x)}{A(x)}\right)^{\prime}=\frac{2 J(x) K(x)^{2}}{A(x)}$

where, for convenience, we have defined the dimensionless parameter

$\gamma_{0} \equiv-\frac{\Lambda}{3 e^{2} h_{0}^{2}}$ 
and

$$
\begin{aligned}
& V_{1}=K^{\prime}(x)^{2}+\frac{x^{2}}{2} H^{\prime}(x)^{2} \\
& V_{2}=\frac{\left(K(x)^{2}-1\right)^{2}}{2 x^{2}}+\frac{\lambda}{4 e^{2}} x^{2}\left(H(x)^{2}-1\right)^{2}
\end{aligned}
$$

\subsection{The boundary conditions}

Ansatz (11) will lead to well behaved solutions for the matter fields if, at $x=0$, one imposes

- $H(x) / x$ and $J(x) / x$ are regular;

- $1-K(x)$ and $K^{\prime}(x)$ go to zero.

- $\mu(x) \rightarrow 1$

On the other hand we want the system to go asymptotically to anti-de Sitter space which corresponds to the solution of the Einstein equations with $\Lambda<0$ in absence of matter (see next Section ); for this to happen we must impose that the matter energy-momentum tensor vanishes at spatial infinity. From Eq. (10) one can see that the appropriate conditions for $x \rightarrow \infty$ are

$A(x) \rightarrow 1$

$K(x) \rightarrow O\left(x^{-\alpha_{1}}\right)$

$H(x) \rightarrow H_{\infty}+O\left(x^{-1-\alpha_{2}}\right)$

$J(x) \rightarrow J_{\infty}+O\left(x^{-\alpha_{3}}\right)$

with $\alpha_{i}>0, i=1,2,3$.

Note that, being the equations for $A$ and $\mu$ first order, we impose just one condition for each one.

\section{The system in AdS space}

We shall first consider the case in which the Newton constant $G$ vanishes, so that the gravitational equations decouple from the matter and then analyse the full $G \neq 0$ problem. In the former case we have already studied in [18] the classical equations of motion analytically, showing that monopoles could exist in asymptotically anti-de Sitter spaces and discussed its main properties. We present in the next subsection the numerical evidence that this solutions do exist, thus completing the analysis in [18].
Then, we extend our study to the $G \neq 0$ case and again present both analytical and numerical analysis showing the existence of monopole solutions provided $G$ is smaller than a critical value $G_{c}$.

\subsection{The $G=0$ case}

Taking the $\alpha_{0} h_{0}{ }^{2} \rightarrow 0$ limit, one easily finds for the metric the solution

$A(x)=1$

$\mu(x)=1+\gamma_{0} x^{2}-\frac{a}{x}$

which is nothing but the vacuum solution of the Einstein equations with a cosmological constant (assumed negative), and corresponds to a neutral Schwarzschild black hole in AdS space. Concerning the integration constant $a$, it is related to the mass of the black hole and will be put to zero in what follows, in agreement with the condition imposed on $\mu$ at $x=0$. This metric, in turn, acts as a (AdS) background with radius $r_{0}$,

$r_{0}=\sqrt{-3 / \Lambda}$

for the Yang-Mills-Higgs system.

For simplicity we study Eq. (16) in the BPS limit which corresponds to $\lambda / e^{2}=0$ with $h_{0}$ fixed.

$$
\begin{aligned}
& \left(\mu(x) K^{\prime}(x)\right)^{\prime} \\
& \quad=K(x)\left(\frac{K(x)^{2}-1}{x^{2}}+H(x)^{2}-\frac{J(x)^{2}}{\mu(x)}\right) \\
& \left(x^{2} \mu(x) H^{\prime}(x)\right)^{\prime}=2 H(x) K(x)^{2} \\
& \mu(x)\left(x^{2} J^{\prime}(x)\right)^{\prime}=2 J(x) K(x)^{2}
\end{aligned}
$$

The total amount of matter $M$ associated to the solution of (22) is defined as (see for example [13])

$M=\int_{\Sigma_{t}} d^{3} x \sqrt{g^{(3)}} T_{00}$

where $g^{(3)}$ is the determinant of the induced metric on surfaces $\Sigma_{t}$ of constant time $t$ with normal vector $e_{0}=\mu(x)^{-1 / 2} \partial_{t}$ and $T_{00} \equiv e_{0}^{\mu} e_{0}^{\nu} T_{\mu \nu}=T_{t t} / \mu(x)$ is the local energy density as seen by an observer moving on the flux lines of $\partial_{t}$. For the spherically 
symmetric configuration we are considering, it takes the form

$$
M=\frac{4 \pi h_{0}}{e} \int_{0}^{\infty} d x \frac{x^{2}}{\left(1+\gamma_{0} x^{2}\right)^{\frac{3}{2}}} \frac{T_{t t}}{e^{2} h_{0}^{4}}
$$

We quote for completeness the explicit expressions for $T_{t t}=T_{t t}^{(\mathrm{YM})}+T_{t t}^{(\mathrm{H})}$

$$
\begin{aligned}
\frac{T_{t t}^{(\mathrm{YM})}}{e^{2} h_{0}{ }^{4}}= & \frac{\mu(x)}{2} J^{\prime}(x)^{2}+\frac{J(x)^{2} K(x)^{2}}{x^{2}} \\
& +\frac{\mu(x)^{2} K^{\prime}(x)^{2}}{x^{2}} \\
& +\frac{\mu(x)}{2 x^{4}}\left(K(x)^{2}-1\right)^{2} \\
\frac{T_{t t}^{(\mathrm{H})}}{e^{2} h_{0}{ }^{4}}= & \frac{\mu(x)^{2}}{2} H^{\prime}(x)^{2}+\frac{\mu(x)}{x^{2}} H(x)^{2} K(x)^{2}
\end{aligned}
$$

It is not difficult to see from these expression that the boundary conditions imposed through Eq. (19) are precisely those required for finiteness of $M$.

As stated above, analytical arguments showing the possibility of monopole solutions were presented in [18]. To begin with, let us note that is possible to perform a power series expansion for large $x$ and calculate the coefficients in the expansion recursively. One can consistently propose an expansion of the form

$$
\begin{aligned}
K(x)= & \frac{K_{\nu+1}}{x^{\nu+1}}\left(1-\frac{k_{\nu}}{x^{2}}+\ldots\right) \\
H(x)= & \frac{3 H_{3}}{x^{3}} \sum_{k=0}^{\nu} \frac{(-)^{k}}{2 k+3} \frac{1}{x^{2 k}} \\
& +H_{\infty}\left(1+\frac{h_{\nu}}{x^{2 \nu+4}}+\ldots\right) \\
J(x)= & \frac{J_{1}}{x}+J_{\infty}\left(1+\frac{j_{\nu}}{x^{2 \nu+4}}+\ldots\right)
\end{aligned}
$$

and, after insertion in Eq. (22), one can determine the coefficients $k_{\nu}, h_{\nu}$ and $j_{\nu}$ recursively. For positive integer $\nu$ one has

$$
k_{\nu}=\frac{\nu^{2}+3 \nu+3+J_{\infty}^{2}}{2(2 \nu+3)}
$$

$$
\begin{aligned}
& h_{\nu}=\frac{K_{\nu+1}^{2}}{(\nu+2)(2 \nu+1)} \\
& j_{\nu}=\frac{K_{\nu+1}^{2}}{(\nu+2)(2 \nu+3)}
\end{aligned}
$$

Similar expressions can be obtained for $\nu$ a positive semi-integer.

When such an expansion are assumed, non-trivial solutions exist if and only if

$H_{\infty}^{2}=\nu(\nu+1) \gamma_{0}, \quad \nu=\frac{1}{2}, 1, \frac{3}{2}, 2, \ldots$

If one relaxes a power behavior like in (26), then one again gets a relation like (28) but with $\nu$ a real number, the leading exponent in the asymptotic expansion of $K(x)$. It is important to note that, having AdS space a natural scale $r_{0}$, the system trades the in principle arbitrary $h_{0}$ dimensionfull parameter for the AdS radius $r_{0}=\sqrt{-3 / \Lambda}$ which now sets the scale,

$$
|\boldsymbol{H}(\infty)|^{2}=\nu(\nu+1) \gamma_{0} h_{0}^{2}=\nu(\nu+1)\left(e r_{o}\right)^{-1}
$$

Then, using (28) for $\nu$ real and a given $\Lambda$ is equivalent to consider an integer $\nu$ (for example with $\nu=1$ ) provided $r_{0}$ (i.e. $\Lambda$ ) is changed accordingly.

To obtain a detailed profile of the monopole solution, we solved numerically the differential equations. For simplicity we considered the $J=0$ case corresponding to a purely magnetic solution. The equations of motion read

$$
\left(\mu(x) K^{\prime}(x)\right)^{\prime}=K(x)\left(\frac{K(x)^{2}-1}{x^{2}}+H(x)^{2}\right)
$$

$\left(x^{2} \mu(x) H^{\prime}(x)\right)^{\prime}=2 H(x) K(x)^{2}$

$\mu(x)=1+\gamma_{0} x^{2}$

We employed a relaxation method for boundary value problems [19]. Such method determines the solution by starting with an initial guess and improving it iteratively. The natural initial guess was the exact Prassad-Sommerfield solution [20] (which corresponds to $\gamma_{0}=0$ ). We have found regular monopole solutions for any value of the cosmological constant $\Lambda$. We present in Fig. 1 the solution profile for different values of $\Lambda$. A distinctive fea- 


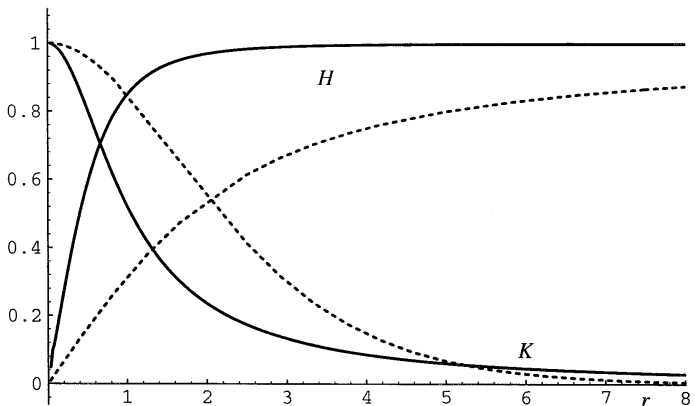

Fig. 1. Plot of the functions $K(r)$ and $H(r)$ (in dimensionless variables) for the monopole solution with $\lambda=0$ and $\alpha_{0}=0$. The solid line corresponds to the solution with $\gamma_{0}=1.0$ and the dashed line corresponds to the BPS flat space solution.

ture of solutions for $\Lambda \neq 0$ compared with the flatspace Prasad-Sommerfield solution concerns the asymptotic behavior of the fields. Indeed, when $\Lambda \neq$ 0, the Higgs field approaches its v.e.v. faster than in the Prasad-Sommerfield (PS) case,

$H(x) \sim H_{\infty}+\frac{C_{\Lambda \neq 0}}{x^{3}}, \quad x \gg 1$

$H^{\mathrm{PS}}(x) \sim H_{\infty}-\frac{1}{x}, \quad x \gg 1$

As a result of this change of the asymptotic behavior, one can see that the radius $R_{c}$ of the monopole core decreases. Indeed, as can bee seen in Fig. 1, when $\gamma_{0}$ (the cosmological constant) grows, $R_{c}$ becomes smaller as the magnetic field concentrates near the origin.

We have also computed numerically the monopole mass which can be written as

$M=\frac{4 \pi}{\mathrm{e}^{2}} \frac{1}{r_{0}} f_{\gamma_{0}}\left(\lambda / \mathrm{e}^{2}\right)$

where, extending the usual flat space notation, we have introduced the dimensionless function $f_{\gamma_{0}}\left(\lambda / \mathrm{e}^{2}\right)$. In the present case, the cosmological constant provides a natural scale and this has been exploited in (35). This formula can be written in units of the mass scale $h_{0}$ as

$\frac{M}{h_{0}}=\frac{4 \pi}{e} E$

where $E$ is a dimensionless function of $\gamma_{0}$,

$E=\sqrt{\gamma_{0}} f_{\gamma_{0}}\left(\lambda / \mathrm{e}^{2}\right)$

We present in Fig. 2 a plot for $E$ as a function of $\gamma_{0}$ where one can see that $\lim _{\gamma_{0} \rightarrow 0} \sqrt{\gamma_{0}} f_{\gamma_{0}}(0)=1$ which is the correct result for Prasad-Sommerfield monopoles in flat space.

\subsection{The $G \neq 0$ case}

In order to study the asymptotic behavior of the solutions to Eq. (13) we have taken as independent metric functions $A(x)$ and $\tilde{\mu}(x)=A(x) \mu(x)$. Moreover, identifying $h_{0}=\left(e r_{0}\right)^{-1}\left(\gamma_{0}=1\right)$, the equations to study become, in the BPS limit,

$$
\begin{aligned}
& A(x)(x \tilde{\mu}(x))^{\prime}=\left(1+3 x^{2}\right) A(x)^{2} \\
& -\alpha_{0} h_{0}{ }^{2}\left(A(x)^{2} V_{2}\right. \\
& \left.+\frac{x^{2}}{2} J^{\prime}(x)^{2}\right) \\
& x \tilde{\mu}(x)^{2} A^{\prime}(x)=\alpha_{0} h_{0}{ }^{2}\left(\tilde{\mu}(x)^{2} V_{1}\right. \\
& \left.+J(x)^{2} K(x)^{2}\right) A(x) \\
& \left(\tilde{\mu}(x) K^{\prime}(x)\right)^{\prime}=K(x)\left(A(x) \frac{K(x)^{2}-1}{x^{2}}\right. \\
& \left.+A(x) H(x)^{2}-\frac{J(x)^{2}}{\tilde{\mu}}\right) \\
& \left(x^{2} \tilde{\mu}(x) H^{\prime}(x)\right)^{\prime}=2 A(x) H(x) K(x)^{2} \\
& \tilde{\mu}(x) A(x)\left(x^{2} J^{\prime}(x)\right)^{\prime}=x^{2} \tilde{\mu}(x) A^{\prime}(x) J^{\prime}(x) \\
& +2 A(x)^{2} K(x)^{2} J(x)
\end{aligned}
$$

where, in the first one, we have combined Eqs. (12) and (13).

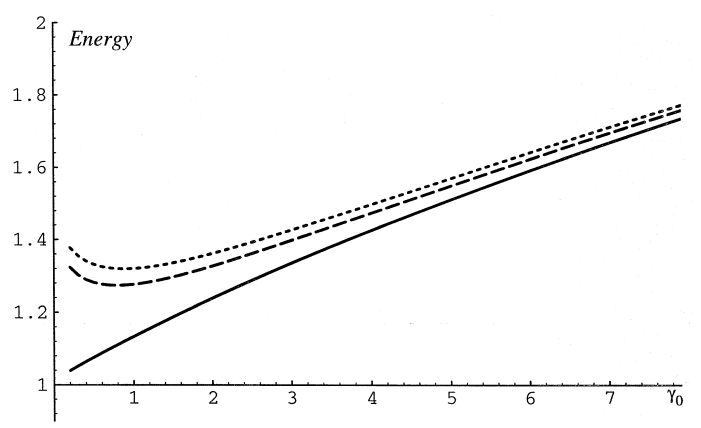

Fig. 2. Energy of the monopole configuration as a function of $\gamma_{0}$ for $h_{0}=1$ and different values of $\lambda: \lambda=0$ (solid line), $\lambda=10$ (dashed line) and $\lambda=20$ (dotted line) in the $\alpha_{0}=0$ case. 
We consider for simplicity the purely magnetic case, $J=0$, and propose a power series expansion of the form

$$
\begin{aligned}
& \tilde{\mu}(x)=x^{2}+\sum_{m=0}^{\infty} \frac{\tilde{\mu}_{m}}{x^{m}}, \quad \tilde{\mu}_{0} \equiv 1 \\
& A(x)=\sum_{m=0}^{\infty} \frac{A_{m}}{x^{m}}, \quad A_{0} \equiv 1 \\
& K(x)=\left\{\begin{array}{l}
\sum_{m=0}^{\infty} \frac{K_{m}}{x^{m}}, \quad K_{0} \equiv 0 \\
\frac{1}{\sqrt{x}} \sum_{m=0}^{\infty} \frac{K_{m}}{x^{m}} \\
H(x)=\sum_{m=0}^{\infty} \frac{H_{m}}{x^{m}}, \quad H_{0} \equiv H_{\infty}, H_{1} \equiv 0
\end{array}\right.
\end{aligned}
$$

Again, coefficients can be determined recursively. The leading coefficients in the expansions for $K$ and $H$ coincide with those already presented for $\alpha_{0}=0$ (Eq. (26)). We then just quote the corresponding ones for the metric functions (for $\nu$ a positive integer)

$$
\begin{aligned}
& \tilde{\mu}(x)=1+x^{2}+\frac{\tilde{\mu}_{1}}{x}+\frac{\alpha_{0} h_{0}^{2}}{2 x^{2}}-\frac{A_{6}}{x^{4}}+\ldots \\
& A(x)=1+\frac{A_{6}}{x^{6}}+\ldots
\end{aligned}
$$

where

$$
A_{6}=-\alpha_{0} h_{0}{ }^{2}\left(\frac{3}{4} H_{3}{ }^{2}+\frac{2}{3} K_{2}^{2} \delta_{\nu, 1}\right)
$$

We see that function $A$ is completely determined, to all orders, in terms of $H$ and $K$ coefficients. As an example, and from the numerical results described below, one finds for $\nu=1$ and $\alpha_{0}=0.1$ that $\tilde{\mu}_{1}=$ $-0.24, K_{2}=0.73, H_{3}=-0.32$ and then $A_{6}=$ -0.87 .

Concerning the asymptotic expansion for $\tilde{\mu}$ corresponds to a Reissner-Nördstrom metric (with cosmological constant), with the free parameter $-\tilde{\mu}_{1}$ related to the gravitatory mass and the coefficient of the $1 / x^{2}$ term, which arises for charged solutions, precisely corresponding to the $Q_{m}=1$ magnetic solution we are considering. As seen from afar, and for an appropriate set of parameters, the metric can be identified with that of a magnetically charged black hole as that described in $[18,21]$. Concerning the expansion for $K$, it has one free coefficient $\left(K_{\nu+1}\right)$, while for the expansion for $H$ two coefficients remain free $\left(H_{\infty}\right.$ and $\left.H_{3}\right)$.

In order to get the detailed profile of the solutions, we have again to solve numerically the equations of motion. For simplicity, we have considered the BPS limit, $\lambda / \mathrm{e}^{2}=0$. Employing the same relaxation method as for the $G=0$ case we have found a self-gravitating monopole solution satisfying the boundary conditions previously discussed. Solutions are similar to those corresponding to asymptotically flat space [6-12]. In particular, we have found a maximum value for the gravitational interaction strength $\alpha_{0}$ such that above $\alpha_{0}^{c}$ the solution ceases to exist. This effect, already encountered in asymptotically flat space, can be understood noting that as $\alpha_{0}$ increases from 0 to its critical value, the ratio $\mathscr{M}=$ mass /radius for the monopole solution also increases until it becomes gravitationally unstable. Now, as the cosmological constant $|\Lambda|$ increases, the radius of the monopole decreases (the behavior for $\alpha_{0} \neq 0$ is analogous to that depicted in Fig. 1 for $\alpha_{0}=0$ ) while the mass of the monopole increases (the behavior for $\alpha_{0} \neq 0$ is analogous to that in Fig. 2) so that $\mathscr{M}$ is a monotonically growing function of $-\Lambda$ or, what is the same, of $\gamma_{0}$. This explains why the critical value $\alpha_{0}^{c}\left(\gamma_{0}\right)$ for $\gamma_{0}>0$, is smaller than the asymptotically flat one, $\alpha_{0}^{c}\left(\gamma_{0}\right)<\alpha_{0}^{c}(0)$ : the critical value $\mathscr{M}_{c}$ at which the solution collapses is

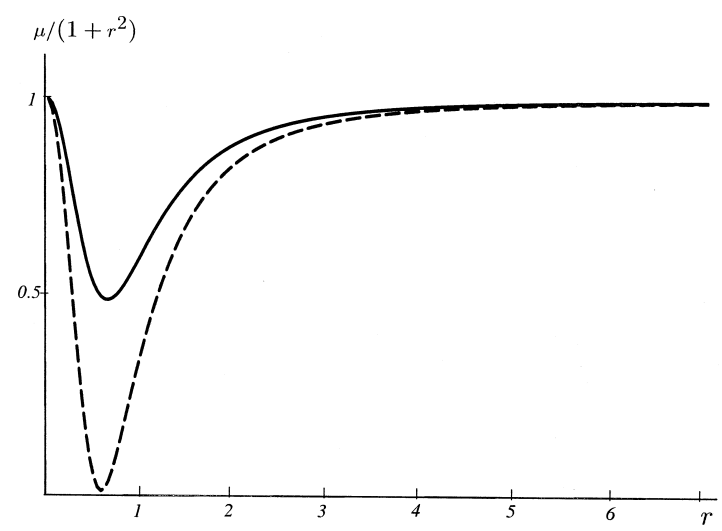

Fig. 3. The solution for the metric function $\mu(r) /\left(1+r^{2}\right)$ for fixed $\gamma_{0}=1$ and $\lambda=0$. The solid line corresponds to $\alpha_{0}=1$ and the dashed one to $\alpha_{0}=\alpha_{0}^{c} \approx 1.371$. 


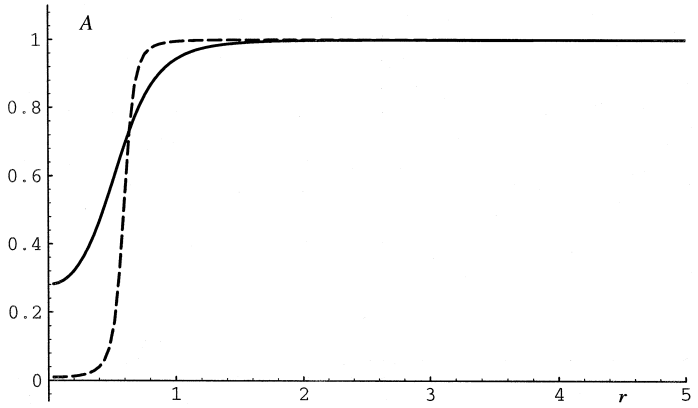

Fig. 4. The solution for the metric function $A(r)$ for fixed $\gamma_{0}=1$ and $\lambda=0$. The solid line corresponds to $\alpha_{0}=1$ and the dashed one to $\alpha_{0}=\alpha_{0}^{c}$.

reached before, in the $\alpha$ domain, for $\gamma_{0}>0$ than for $\gamma_{0}=0$. As an example, for $\lambda=0$ and $\gamma_{0}=1$ the critical $\alpha_{0}$-value is $\alpha_{0}^{c}(1)=1.374$ to be compared with the asymptotically flat space value $\alpha_{0}^{c}(0)=$ 5.549 .

Concerning the solution for the metric, as can be seen in Fig. 3, $\mu /\left(1+\gamma_{0} x^{2}\right)$ has a minimum which decreases as the strength of the gravitation interaction grows and tends to zero as $\alpha_{0} \rightarrow \alpha_{0}^{c}$. A similar behavior can be seen to occur for $A(x)$, see Fig. 4, which has also a minimum at the origin which tends to zero as $\alpha_{0} \rightarrow \alpha_{0}^{c}$. As in the case of asymptotically flat space, $A$ develops a step-like behavior which becomes more and more sharp as $\alpha_{0}^{c}$ is approached. The position of the center of the step function can be used to determine the corresponding value of the horizon, which for AdS spaces results from the solution of a quartic algebraic equation. [21,18]

\section{Discussion}

In this work we have studied in detail the monopole and dyon solutions to Yang-Mills-Higgs theory coupled to gravity for asymptotically anti-de Sitter space presented in Ref. [18]. We have first considered the case in which the Newton constant $\alpha_{0}$ vanishes so that the Einstein equations decouple leading to a Schwarzschild black hole in AdS space. This metric acts as a background for dyon solutions which were studied in detail making both an analytical and a numerical analysis. A distinctive feature of
AdS solutions in this case is that the monopole radius is smaller than that corresponding to the $\Lambda=0$ case. Apart from this property, qualitatively, the Higgs field and magnetic field behavior is very similar to that corresponding to the 't Hooft-Polyakov solution. More interesting is the behavior of solutions where gravity is effectively coupled to the matter fields. In first place, as it happens in asymptotically flat space, a critical value for the Newton constant exists above which no regular monopole or dyon solution can be found. This effect was explained in asymptotically flat spaces [4-15] by noting that as $\alpha_{0}$ grows the mass of the monopole grows and its radius decreases so that it finally becomes gravitationally unstable. Now, the presence of a cosmological constant enhances this effect and for this reason, the critical value we find, $\alpha_{0}^{c}(\Lambda)$ is smaller than the asymptotically flat one.

\section{Acknowledgements}

This work is partially supported by CICBA, CONICET (PIP 4330/96), ANPCYT (PICT $97 / 2285)$.

\section{References}

[1] F.A. Bais, R.J. Russel, Phys. Rev. D 115 (1975) 2692.

[2] Y.M. Cho, P.G.O. Freund, Phys. Rev. D 12 (1975) 1588.

[3] P. van Nieuwenhuizen, D. Wilkinson, M.J. Perry, Phys. Rev. D 13 (1976) 778.

[4] K. Lee, V.P. Nair, E.J. Weinberg, Phys. Rev. D 45 (1992) 2751.

[5] M.E. Ortiz, Phys. Rev. D 45 (1992) R2586.

[6] P. Breitenlohner, P. Forgács, D. Maison, Nucl. Phys. B 383 (1992) 357.

[7] B.S. Balakrishna, K.C. Wali, Phys. Rev. D 46 (1992) R5228.

[8] A.V. Nguyen, K.C. Wali, Phys. Rev. D 51 (1995) 1664.

[9] P. Breitenlohner, P. Forgács, D. Maison, Nucl. Phys. B 442 (1995) 126.

[10] Y. Brihaye, B. Hartmann, J. Kunz, Phys. Lett. B 441 (1998) 77.

[11] Y. Brihaye, B. Hartmann, J. Kunz, N. Tell, Phys. Rev. D 60 (1999) 104016.

[12] A. Lue, E.J. Weinberg, Phys. Rev. D 60 (1999) 084025.

[13] G.W. Gibbons, Lectures given at 12th Lisbon Autumn School on Physics, Lisbon, Portugal, Oct 1-5, 1990. Published in Lisbon Autumn School 1990:110-133.

[14] D. Maison, to be published in the Proc. of Pacific Conference 
on Gravitation and Cosmology, Seoul, Korea, 1996, grqc/9605053 report.

[15] M.S. Volkov, D.V. Gal'tsov, Phys. Reports C 319 (1998) 77.

[16] E. Winstanley, Class. Quant. Grav. 16 (1999) 1963.

[17] J. Bjoraker, Y. Hosotani, gr.qc/9906091 report.

[18] A.R. Lugo, F.A. Schaposnik, hep-th/9909226 report, Phys. Lett. B, in press.
[19] W.H. Press, S.A. Teukolsky, W.T. Vetterling, Numerical Recipes: The art of Scientific Computing, Cambridge University Press, 1992.

[20] M.K. Prasad, C.M. Sommerfield, Phys. Rev. Lett. 35 (1975) 760.

[21] L.J. Romans, Nucl. Phys. B 383 (1992) 395. 